\title{
Protective Effect of Anthocyanin on Neurovascular Unit in Cerebral Ischemia/Reperfusion Injury in Rats
}

OPEN ACCESS

Edited by:

Robin Polt,

The University of Arizona,

United States

Reviewed by:

Hong Zhang,

The University of Arizona,

United States

Luigia Trabace,

University of Foggia, Italy

*Correspondence:

Yang Pan

ypan@njucm.edu.cn

${ }^{t}$ These authors have contributed

equally to this work

Specialty section:

This article was submitted to

Neuropharmacology,

a section of the journal

Frontiers in Neuroscience

Received: 28 August 2018 Accepted: 29 November 2018 Published: 11 December 2018

Citation:

Pan Z, Cui M, Dai G, Yuan T, Li Y, Ji T and Pan Y (2018) Protective Effect of Anthocyanin on

Neurovascular Unit in Cerebral Ischemia/Reperfusion Injury in Rats.

Front. Neurosci. 12:947.

doi: 10.3389/fnins.2018.00947

\author{
Zihao Pan ${ }^{1 \dagger}$, Mengdi Cui ${ }^{2 t}$, Guoliang Dai ${ }^{3}$, Tianjie Yuan', Yuhua Li', Tuo Ji' and \\ Yang Pan ${ }^{1 *}$
}

\begin{abstract}
${ }^{1}$ College of Pharmacy, Nanjing University of Chinese Medicine, Nanjing, China, ${ }^{2}$ Thyroid and Breast Surgery, The Third Affiliated Hospital of Nanjing University of Chinese Medicine, Nanjing, China, ${ }^{3}$ Department of Clinical Pharmacology, The Affiliated Hospital of Nanjing University of Chinese Medicine, Nanjing, China
\end{abstract}

Treating cerebral ischemia continues to be a clinical challenge. Studies have shown that the neurovascular unit (NVU), as the central structural basis, plays a key role in cerebral ischemia. Here, we report that anthocyanin, a safe and natural antioxidant, could inhibit apoptosis and inflammation to protect NVU in rats impaired by middle cerebral artery occlusion/reperfusion (MCAO/R). Administration of anthocyanin significantly reduced infarct volume and neurological scores in MCAO/R rats. Anthocyanin could also markedly ameliorate cerebral edema and reduce the concentration of Evans blue (EB) by inhibiting MMP-9. Moreover, anthocyanin alleviated apoptotic injury resulting from $\mathrm{MCAO} / \mathrm{R}$ through the regulation of $\mathrm{Bcl}-2$ family proteins. The levels of inflammationrelated molecules including tumor necrosis factor- $\alpha$ (TNF- $\alpha$ ), interleukin-1 $\beta(\mathrm{IL}-1 \beta)$, and interleukin-6 (IL-6), which were over-expressed with MCAO/R, were decreased by anthocyanin. In addition, Nuclear factor-kappa B (NF-kB) and the NLRP3 inflammasome pathway might be involved in the anti-inflammatory effect of anthocyanin. In conclusion, anthocyanin could protect the NVU through multiple pathways, and play a protective role in cerebral ischemia/reperfusion injury.

Keywords: cerebral ischemia/reperfusion injury, neurovascular unit, anthocyanin, apoptosis, inflammation

\section{INTRODUCTION}

Treating cerebral ischemia continues to be a clinical challenge and underlying mechanisms of cerebral ischemia remain elusive. Recent insight into the basic mechanism involved in cerebral ischemia indicates that the neurovascular unit (NVU) is a new target for the overall study of neuronal damage and protection mechanisms (Lake et al., 2017), which consists of neurons and blood-brain barrier (BBB), including astrocytes, microglia, vascular endothelial cells and extracellular matrix (ECM; Maki et al., 2013). The destruction of the BBB promotes the development of neurological dysfunction in cerebral ischemia (Rosenberg, 2012). MMP-2 and MMP-9, which are the most important ECM degrading enzymes in vivo, are most closely related to the destruction of the BBB (Turner and Sharp, 2016). The use of MMP-9 inhibitors can significantly reduce cerebral edema and cerebral infarct volume caused by ischemia, and reduce BBB damage (Turner and Sharp, 2016).

Apoptosis and the inflammatory response are important processes that cause NVU destruction, and they play a key role in the pathology of stroke (Vidale et al., 2017). In stroke cases, apoptosis occurs mainly in the ischemic penumbra. Activation of apoptosis signaling pathways results in 
excessive oxygen free radical generation, DNA damage, death receptor ligation, and lysosomal protease activation. The mitochondrial apoptotic pathway is one of the predominant pathways of apoptosis (Watts et al., 2013). Studies have found that a variety of regulatory gene products involved in the process of apoptosis, including the cysteine protease (Caspases) family, Bcl-2 family, and p53 (Elmore, 2007). NF-кB and inflammasomes play an important role in the inflammatory cascade (Fann et al., 2017). Recent studies have demonstrated that in response to cerebral ischemia, NLRP3 inflammasomes promote inflammatory responses in neurons and glial cells, resulting in tissue damage (Gao et al., 2017). Increased NLRP3 is also associated with the apoptosis of neurons and glial cells. Studies have shown that activation of the NLRP3 inflammasome in brain cells can regulate the activation of caspase-1, which converts pro-IL- $1 \beta$ into a mature form of IL- $1 \beta$ (Tong et al., 2015). Given these complex mechanisms, the efficacy of a single target drug in the treatment of stroke may not be satisfactory, and, thus, it is necessary to identify a pleiotropic drug.

Anthocyanins are natural flavonoids formed by the combination of anthocyanidin and glycosides, which are widely found in the cytoplasm of flowers, fruits, stems, leaves, and root organs of plants. Studies have confirmed the biological benefits of dietary anthocyanins, as they exhibit anticancer activity, anti-inflammatory activity, neuroprotective activity, anti-obesity activity, anti-diabetic activity, and properties involved in the prevention of cardiovascular disease ( $\mathrm{Li}$ et al., 2017). Anthocyanins can reduce neuronal damage induced by focal cerebral ischemia by blocking the JNK and p53 signaling pathways (Shin et al., 2006). Mechanically, it has been shown that anthocyanins inhibit the NF- $\kappa$ B signaling pathway, mitogenactivated protein kinases, and Cyclooxygenases (COXs; Li et al., 2017). Despite these findings, anthocyanins exhibit a wide range of biological activities, and their mechanisms may be diverse and largely unexplored.

In this study, we investigated the protective effects of anthocyanin in rats with MCAO/R injury, and found some clues on the underlying mechanism of protection.

\section{MATERIALS AND METHODS}

\section{Ethics Statement}

All procedures described in this study were performed in accordance with the guidelines of Nanjing University of Chinese Medicine Animal Care and Use Committee.

\section{Reagents}

Chromatographic grade methanol and acetonitrile were purchased from Merck (Darmstadt, Germany). Formic acid, ethyl acetate and other chemicals were purchased from commercial sources and of analytical grade. Rabbit monoclonal to Bad, rabbit monoclonal to $\mathrm{Bcl}-\mathrm{xl}$, rabbit monoclonal to $\mathrm{Bax}$, rabbit polyclonal to $\mathrm{Bcl}-2$, rabbit polyclonal to $\mathrm{p}-\mathrm{NF}-\kappa \mathrm{B}$ p50, rabbit polyclonal to $\mathrm{p}-\mathrm{NF}-\kappa \mathrm{B}$ p65, and rabbit monoclonal to $\mathrm{p}-\mathrm{I} \kappa \mathrm{B}$ were purchased from Abcam (Cambridge, United Kingdom). Antibodies for mouse monoclonal to NLRP3 were acquired from Adipogen
International (San Diego, CA, United States). Antibody for glyceraldehyde-3-phosphate dehydrogenase (GAPDH) were acquired from Sigma-Aldrich (St. Louis, MO, United States). Evans blue (EB) was purchased from Sigma Chemical Co., Ltd. (St. Louis, MO, United States). All other reagents were obtained from Sigma-Aldrich (St. Louis, MO, United States).

\section{Anthocyanin Preparation}

Anthocyanin, namely pentunidin-3-O-rutinoside ( $p$-coumaroyl)-5-O-glucoside (purity 98.3\%) was extracted and purified from the dried fruits of Lycium ruthenicum Murr.(LRM, sampled from Delingha, Qinhai, China) according to the method proposed by Wang et al. (2014) with slight modification.

Briefly, anthocyanin was extracted twice from the dried fruits of LRM using $1 \%$ formic acid $\left(40^{\circ} \mathrm{C}\right)$ at a ratio of $1: 30$ for 1 h each time. The crude extract of anthocyanins was subjected to liquidliquid extraction, four times by water-saturated ethyl acetate at a ratio of $1: 3$ for $12 \mathrm{~h}$ each time, and was purified using a column $(1 \mathrm{~cm} \times 15 \mathrm{~cm})$ of Amberlite XAD-7HP macroporous adsorption resin (Sigma-Aldrich, St. Louis, MO, United States). Then, two fractions of anthocyanins were isolated from the Amberlite XAD-7HP eluate using a column $(1 \mathrm{~cm} \times 100 \mathrm{~cm})$ of Sephadex LH-20 (GE Healthcare, Chicago, IL, United States). Subsequently, the monomeric anthocyanin of pentunidin-3-Orutinoside ( $p$-coumaroyl)-5-O-glucoside was separated from the second fraction of the Sephadex LH-20 eluate by an Alltech 1500 semi-preparative HPLC (Chicago, IL, United States) and was identified using an AB SCIEX API 4000 ESI-MS/MS (Framingham, MA, United States).

\section{Animals}

Adult male Sprague-Dawley rats weighing 250-300 g were provided by Model Animal Research Center of Nanjing University. All animals were housed five per cage under controlled temperature $\left(22 \pm 2{ }^{\circ} \mathrm{C}\right)$ and with $12 \mathrm{~h}$ light/dark cycle (lights on at 8:00 a.m.). The animals had free access to food and water. Rats were injected intraperitoneally with different doses of anthocyanin $(50,100$, and $200 \mathrm{mg} / \mathrm{kg})$ for 7 days before the induction of middle cerebral artery occlusion.

\section{MCAO/R Model}

The common carotid artery was isolated following rat anesthesia (10\% chloral hydrate, $350 \mathrm{mg} / \mathrm{kg}$, intraperitoneally), and the branches of the right external carotid artery were carefully separated. A 4-0 nylon monofilament suture with a silicon coated tip was advanced to the internal carotid artery to occlude the source of the MCA. In the sham group, this same procedure was performed but no sutures were inserted. After $2 \mathrm{~h}$ of cerebral ischemia, the suture was withdrawn for reperfusion for $24 \mathrm{~h}$.

\section{Evaluation of Neurological Deficits}

Each rat was evaluated for neurological deficits according to Longa's method (Longa et al., 1989), which included the following neurological scores and symptoms: 0 , no obvious neurological symptoms; 1 , the rat's front paw cannot be fully extended; 2 , the 
rat circles to one side while walking; 3 , the rat leans to one side while walking; and 4, rats cannot walk or lose consciousness.

\section{Evaluation of Cognitive Function}

Each rat was evaluated for cognitive function by novel object recognition test, $24 \mathrm{~h}$ before the test, the mice were habituated to the arenas $(50 \mathrm{~cm} \times 50 \mathrm{~cm}$ plastic container) for $5 \mathrm{~min}$ without objects. The day after the mice re-entered the arenas from the same starting point of the arena (facing the bottom left corner) and granted $10 \mathrm{~min}$ to familiarize themselves with the two identical objects. Exactly $1 \mathrm{~h}$ after the familiarization period, the rats are exposed to two different object: one is same with the training phase and another is a novel object. The time that the rats spend to explore the old and novel objects are recorded, calculated the object interaction ratio according to the formula: object interaction ratio $=$ Novel object exploration time/Old object exploration time.

\section{Evaluation of Infarct Volume}

After $2 \mathrm{~h}$ of MCAO and $24 \mathrm{~h}$ of reperfusion, the brain was quickly removed. Infarct volume was then measured by TTC staining, which revealed the severity of cerebral ischemia. The data was expressed as a percentage of the total hemisphere (Lin et al., 1993).

\section{Evaluation of Cerebral Edema}

Brain water content was measured using a dry-wet method. Brain tissue was removed after $24 \mathrm{~h}$ of reperfusion, and was immediately weighed (a) It was then baked in a $120^{\circ} \mathrm{C}$ oven for $24 \mathrm{~h}$ and weighed again (b) Brain water content was measured according to the formula: brain water content $=(a-b) / a \times 100 \%$.

\section{Evaluation of BBB Permeability}

The rats in each group were injected with $2 \% \mathrm{~EB}(2 \mathrm{ml} / \mathrm{kg})$ in the tail vein $2 \mathrm{~h}$ before sacrifice. The brain tissue was then removed and weighed, and then placed into dimethylformamide $\left(1 \mathrm{ml} / 100 \mathrm{mg}\right.$ brain tissue) and incubated at $60^{\circ} \mathrm{C}$. for $24 \mathrm{~h}$. After centrifugation at $1000 \mathrm{r} / \mathrm{min}$ for $5 \mathrm{~min}$, the supernatant was taken and the absorbance at a wavelength of $620 \mathrm{~nm}$ was measured with a spectrophotometer.

\section{Gelatin Zymography}

Protein concentrations were determined after samples collection. The samples, mixed with loading buffer, were loaded into the wells of a precast gel and subjected to electrophoresis. Then the gel was washed in washing solution followed by shaking in incubation solution for $48 \mathrm{~h}\left(37.5^{\circ} \mathrm{C}\right)$. After staining with Coomassie Brilliant Blue, the gel was bleached by different concentrations of methanol-acetic acid.

\section{Serum ELISA Detection}

Samples of $0.5 \mathrm{ml}$ of blood were obtained from the femoral vein at $24 \mathrm{~h}$ after reperfusion. The serum was separated and collected by centrifugation. Then the serum samples were then assessed for inflammatory mediator (TNF- $\alpha$, IL-1 $\beta$, and IL-6) levels using an ELISA kit, according to the manufacturer's instructions.

\section{Western Blot}

Tissues were placed in a pre-cooled glass grinder, a precooled lysate containing PMSF and phosphatase inhibitors was added, and they were thoroughly ground and lysed. An appropriate amount of $5 \mathrm{X}$ protein loading buffer was added to the protein sample. The protein $(50 \mu \mathrm{g})$ was separated by SDS-PAGE and transferred to PVDF membranes. The membranes were blocked with $5 \%$ bovine serum albumin at room temperature for $1 \mathrm{~h}$ and then incubated with a specific primary antibody overnight at $4^{\circ} \mathrm{C}$. The following primary antibodies used: $\mathrm{Bad}(1: 2000)$, Bax (1:1000), Bcl-2 (1:1000), Bcl-

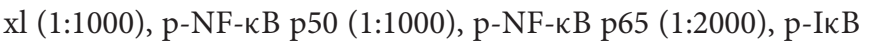
(1:5000), and NLRP3 (1:500). The corresponding secondary antibody was incubated for $1 \mathrm{~h}$ at room temperature and the blot was visualized using the enhanced chemiluminescence (ECL) method. Software Quantity One -4.6.5 software (Bio-Rad Laboratories, Irvine, CA, United States) was used to analyze the data.

\section{Real Time-PCR}

Trizol reagent was added to the sample, which was homogenized, and the RNA was extracted. cDNA was then synthesized and SYBR Green I dye was used for real-time quantitative PCR. The sense and antisense primers used for the analysis of rat TNF- $\alpha$, IL-1 $\beta$, IL-6, and GAPDH expression were as follows. TNF-a: $5^{\prime}$-CCC CTTTATCGTCTA CTCCTC-3' and 5'-GCTGGTAGTTTAGCTCCGTTT- $3^{\prime}$ (553 bp). IL-1b: $5^{\prime}$-TCATTGTGGCTGTGGAGAAG-3' and 5'-CTATGTCCCGACCATTGCTG-3' (579 bp). IL-6: $5^{\prime}$-GGA TACCACCCACAACAGAC- $3^{\prime}$ and 5'-TTGCCGAGTAGAC CTCATAG-3' $3^{\prime}(520$ bp $)$ GAPDH: $5^{\prime}$-CCATCACTGC CACTCAGAAGA-3' ${ }^{\prime}$ and $5^{\prime}$-CATGAGGTCCACCACCCTGT-3' (446 bp).

\section{Histomorphological Analysis}

After the tissue was formed into a block of wax, it was cut into $4 \mu \mathrm{m}$ slices, dewaxed, and hydrated. It was stained with hematoxylin for $4 \mathrm{~min}$ and eosin for $90 \mathrm{~s}$. It was dehydrated with an alcohol gradient from low to high concentrations, made transparent with xylene, and then observed under the microscope (Li et al., 2002).

\section{TUNEL Staining}

The terminal deoxy transferase reaction mixture was incubated with the sections for $1 \mathrm{~h}$ according to the manufacturer's instruction. All of the counted nuclei were stained with hematoxylin.

\section{Statistical Analysis}

Statistical analysis was carried out using ANOVA. Results are expressed as mean \pm SD. A $p$ value $<0.05$ was considered statistically significant. All analyses were performed using GraphPad Prism Version 5.01 software (GraphPad Software Inc., San Diego, CA, United States). 
A

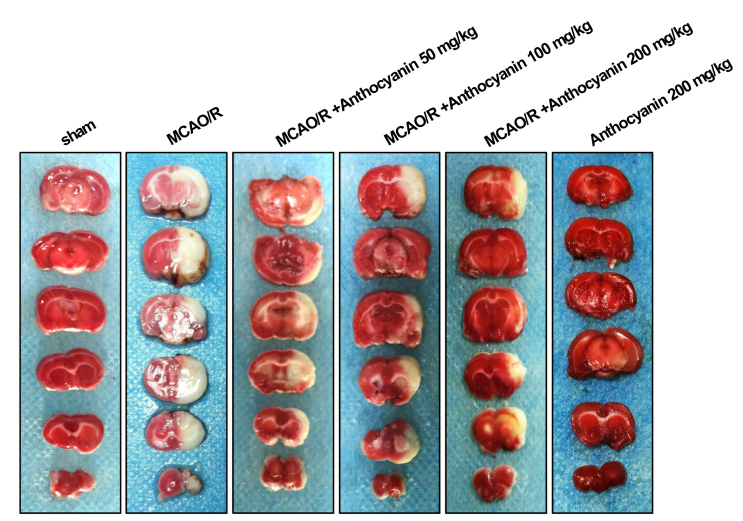

C

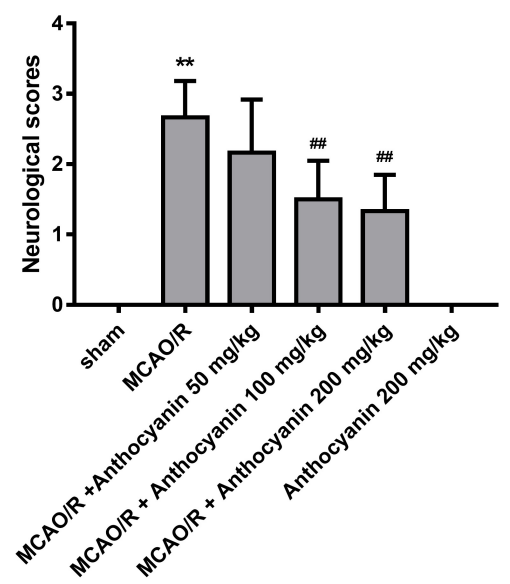

E

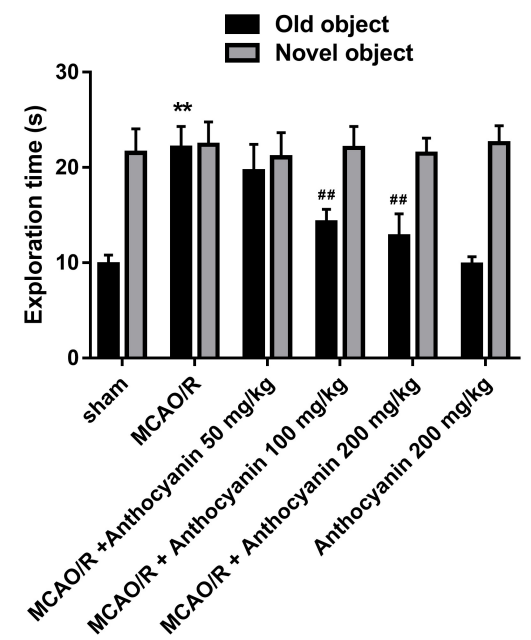

B

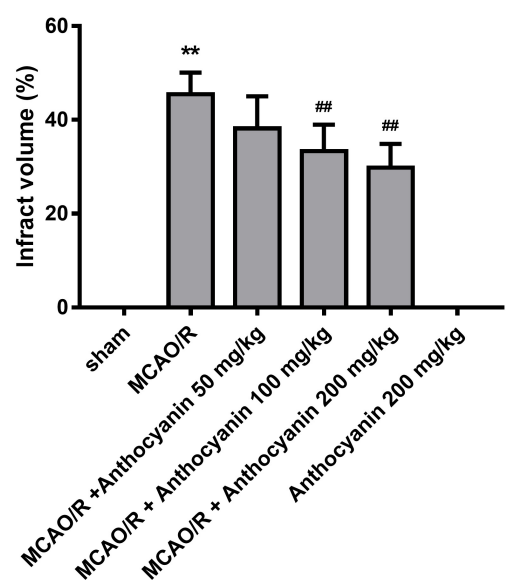

D

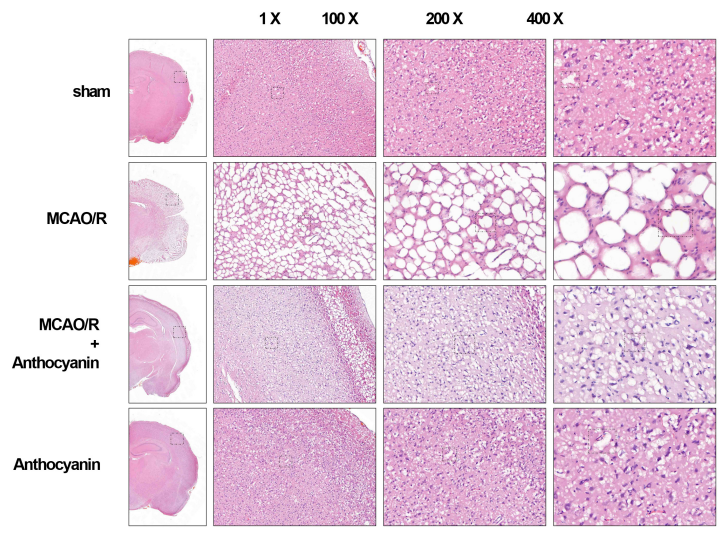

F

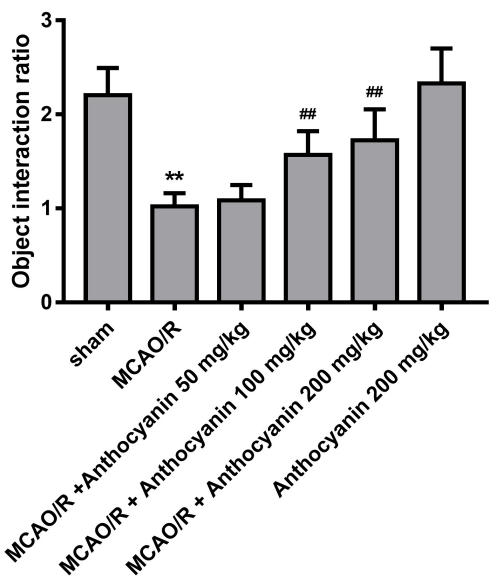

FIGURE 1 | Anthocyanin reduces infarct volume and improves neurological deficits. (A) TTC staining of the brains of rats. (B) Quantitative analysis of infarct volume of rats. $F(5,30)=111.2$. (C) Quantitative analysis of neurological deficits scores in rats. $F(5,30)=31.05$. (D) Hematoxylin and eosin (H\&E) staining of brain sections of rats. (E) The exploration time of novel object recognition test in rats. $F(5,30)=42.84$ (old object). $F(5,30)=0.413$ (novel object). (F) The object interaction ratio of novel object recognition test in rats. $F(5,30)=24.28, n=6,{ }^{* *} p<0.01$ vs. sham; ${ }^{\# \#} p<0.01$ vs. middle cerebral artery occlusion/reperfusion (MCAO/R). 

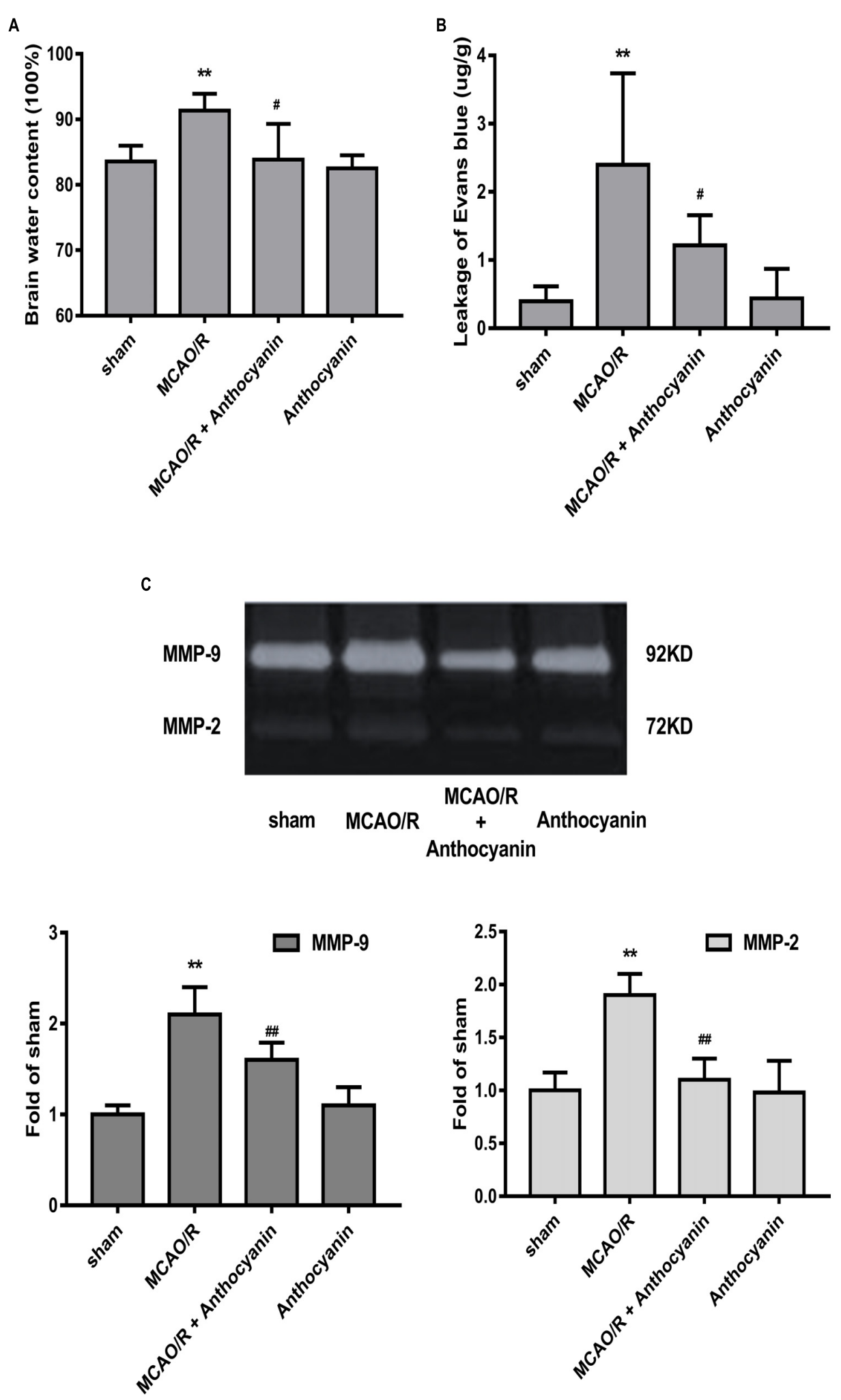

FIGURE 2 | Anthocyanin reduced MCAO/R-induced blood-brain barrier (BBB) destruction by inhibiting MMP-9. (A) Quantitation of brain water contents in rats following MCAO/R. $F(3,20)=8.54$. (B) Quantitative analysis of EB extravasation by spectrophotometry. $F(3,20)=9.473$. (C) Representative band with gelatin zymography and quantitative analysis of the expression levels of MMP-9/2 in rats following MCAO/R. $F(3,20)=49.27(\mathrm{MMP}-9), F(3,20)=24.39(\mathrm{MMP}-2), n=6$, ${ }^{* *} p<0.01$ vs. sham; ${ }^{\#} p<0.05$ and ${ }^{\# \#} p<0.01$ vs. MCAO/R. 


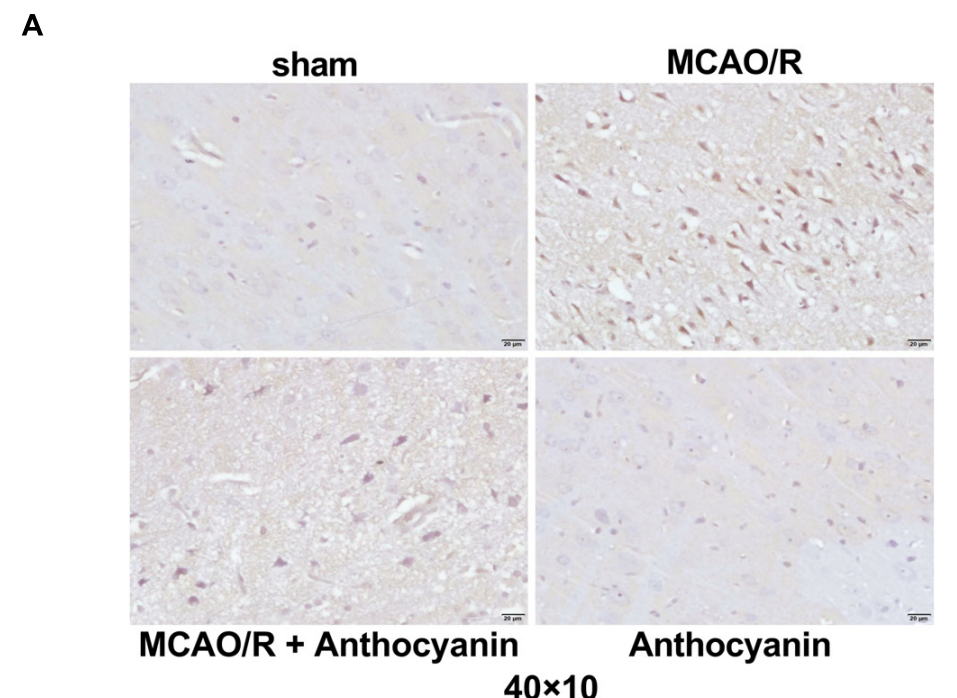

B

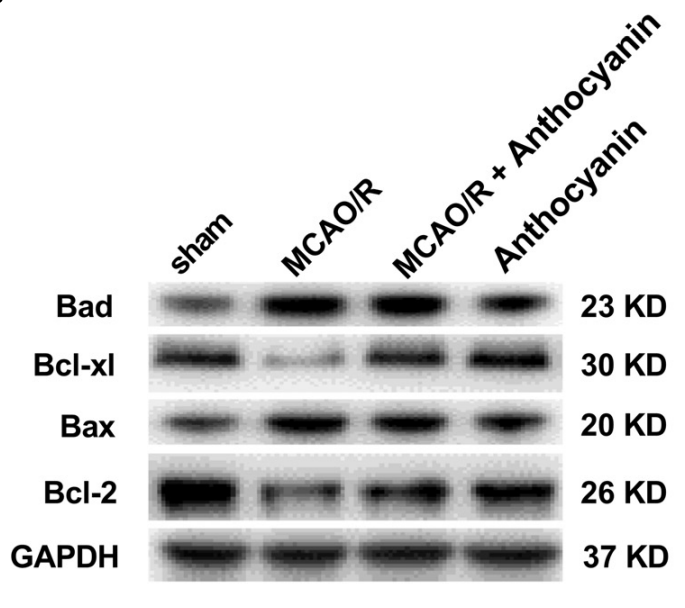

C

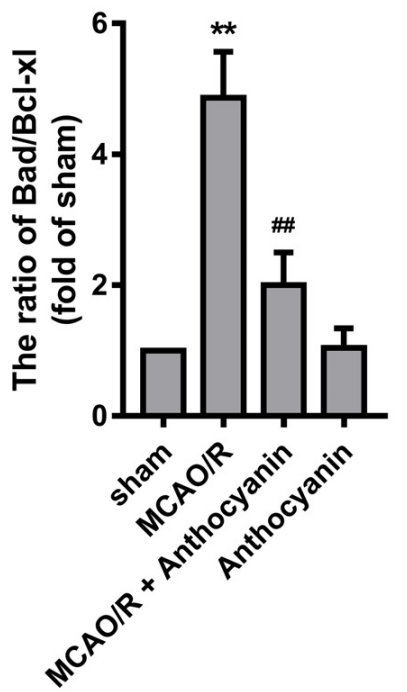

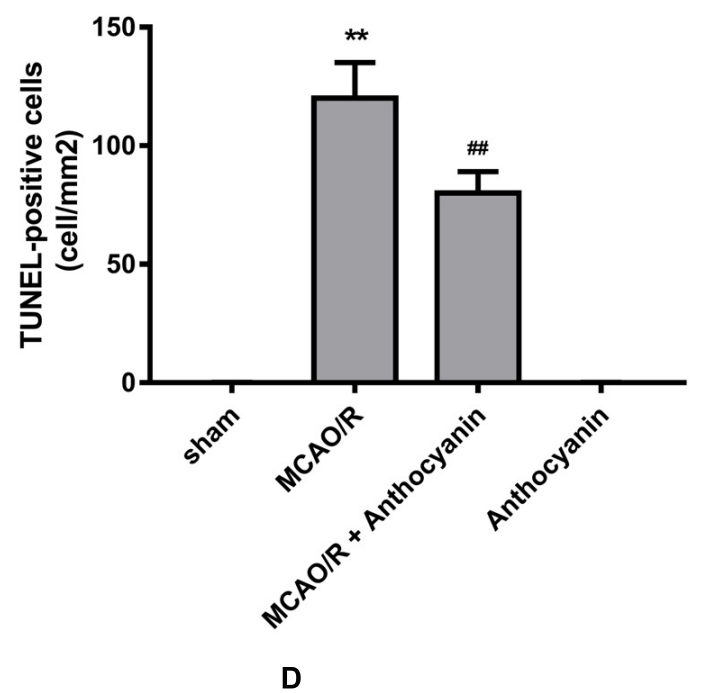

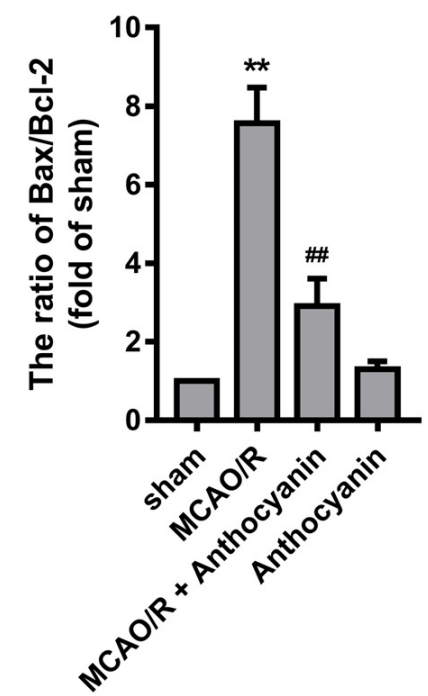

FIGURE 3 | Bcl-2 family proteins are involved in the anti-apoptosis effect of anthocyanin. (A) Representative photomicrographs show TUNEL staining for apoptotic cells in rat brains. Effects on the severity of cerebral apoptosis are shown in an average quantitative analysis of the number of TUNEL-positive cells. $F(3,20)=295$. (B) Western blot analysis of the expression of Bad, Bcl-xl, Bax, and Bcl-2 in rats. (C,D) The expression ratio of Bad/Bcl-xl and Bax/Bcl-2 was quantified by Quantity One 4.6.5 software and is represented as a histogram. $F(3,20)=136.4(\mathrm{Bad} / \mathrm{Bcl}-\mathrm{xl}), F(3,20)=136.7(\mathrm{Bax} / \mathrm{Bcl}-2), n=6,{ }^{* *} p<0.01 \mathrm{vs}$. sham; ${ }^{\#} p<0.01 \mathrm{vs}$. $\mathrm{MCAO} / \mathrm{R}$.

\section{RESULTS}

\section{Anthocyanin Decreased MCAO/R-Induced Brain Infarct Volume and Improved Neurologic Functional Outcomes}

To evaluate the protective effect of anthocyanin, different concentrations of anthocyanin (50, 100, and $200 \mathrm{mg} / \mathrm{kg}$ ) were injected intraperitoneally into rats before the $\mathrm{MCAO} / \mathrm{R}$ surgery. The infarct area of the brain was examined by
2,3,5-Triphenyltetrazolium chloride staining. MCAO/R produced massive infarction, however, in the $\mathrm{MCAO} / \mathrm{R}$ rats that received anthocyanin injections, the infarct volume was found to be significantly reduced in a dosedependent manner (Figures 1A,B). Additionally, behavioral assessment showed that anthocyanin significantly decreased the neurological severity scores and attenuated cognitive function decline measured by novel object recognition test of $\mathrm{MCAO} / \mathrm{R}$ rats in a dose-dependent manner (Figures $\mathbf{1 C}, \mathbf{E}, \mathbf{F}$ ). Therefore, we used anthocyanin $200 \mathrm{mg} / \mathrm{kg}$ for subsequent experiments-the dose that resulted in the greatest effect. 
In addition, as shown in Figure 1D, neuronal loss and the presence of numerous vacuolated spaces were observed in $\mathrm{MCAO} / \mathrm{R}$ rats, which could be ameliorated by anthocyanin (200 mg/kg).

\section{Anthocyanin Inhibited MCAO/R-Induced BBB Destruction by Inhibiting MMP-9}

Blood-brain barrier is the core structure of the NVU, which is damaged in MCAO/R. Therefore, we next examined brain edema and leakage, which is believed to reflect the permeability of the $\mathrm{BBB}$. The results show that cerebral edema in the anthocyanintreated rats was significantly alleviated compared with MCAO/R rats (Figure 2A). Similarly, anthocyanin significantly reduced the leakage of EB content (Figure 2B).

Many studies have confirmed that MMP-9 causes BBB damage in cerebral ischemia (Kurzepa et al., 2014). Therefore, we examined the effect of anthocyanin on MMP-9 levels. Gelatin zymography results showed that $\mathrm{MCAO} / \mathrm{R}$ induced a significant increase in MMP-9, which could be reduced by anthocyanin treatment (200 mg/kg) (Figure 2C).

\section{Mitochondria Are Involved in the} Anti-apoptotic Effect of Anthocyanin

Many studies have reported that the Bcl-2 family is an important apoptosis regulator in the mitochondria (Gross, 2016). A large number of TUNEL-positive cells was observed in the MCAO/R rats, and anthocyanidin (200 $\mathrm{mg} / \mathrm{kg})$ significantly reduced the number of TUNEL-positive cells, as shown in Figure $\mathbf{3 A}$. In addition, anthocyanin significantly increased the $\mathrm{Bcl}-\mathrm{xl} / \mathrm{Bad}$ and $\mathrm{Bcl}-2 / \mathrm{Bax}$ ratios (Figures 3B-D), suggesting that it had attenuated the apoptosis signaling pathway by modulating mitochondrial function.

\section{Anthocyanin Inhibits MCAO/R-Induced Inflammatory Molecule Expression}

Next, we examined the effect of anthocyanin on the inflammatory response. The results showed that compared with the sham rats, $\mathrm{MCAO} / \mathrm{R}$ significantly up-regulated the expression of TNF$\alpha$, IL-1 $\beta$, and IL- 6 in the cerebral cortex, and anthocyanin

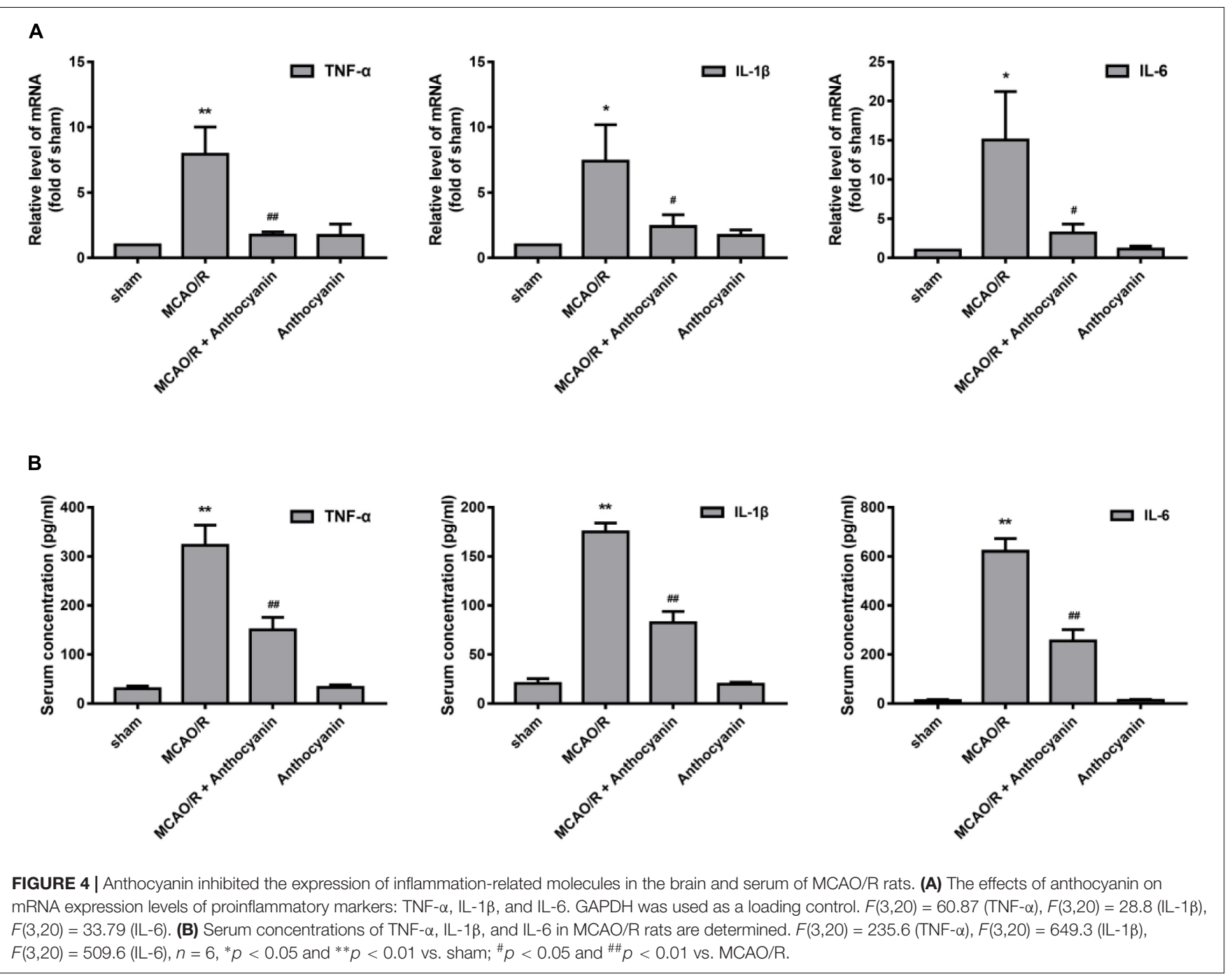




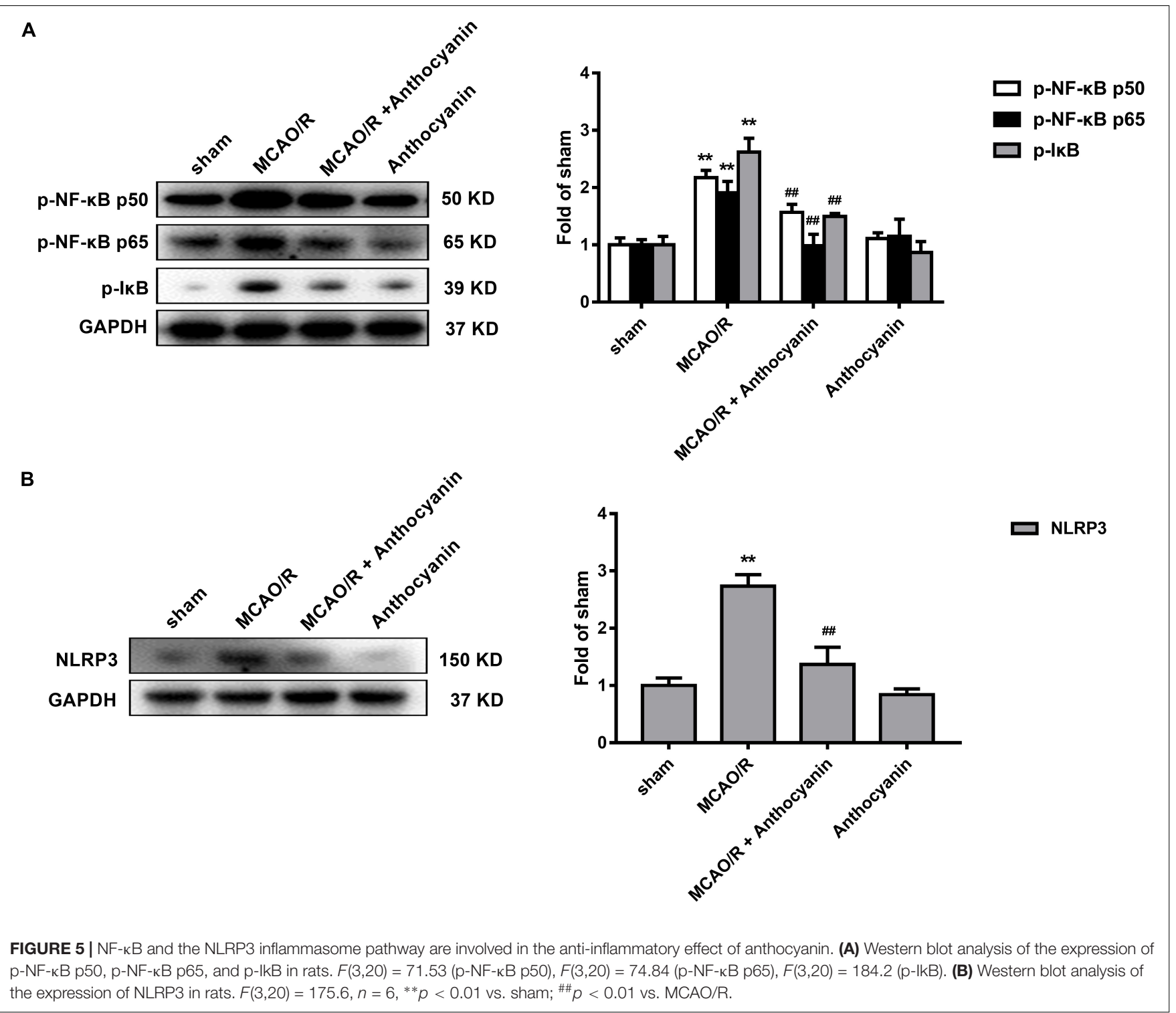

significantly inhibited the expression of TNF- $\alpha$, IL-1 $\beta$, and IL-6 (Figure 4A). We then used ELISA to measure serum TNF- $\alpha$, IL- $1 \beta$, and IL- 6 concentrations. The results showed that anthocyanin significantly reduced $\mathrm{MCAO} / \mathrm{R}$ induced elevated levels of TNF- $\alpha$, IL- $1 \beta$, and IL- 6 , which is consistent with the mRNA results (Figure 4B). These findings suggest that anthocyanin could inhibit the expression of inflammation-related molecules in rat brains, induced by $\mathrm{MCAO} / \mathrm{R}$.

\section{NF- $\kappa$ B and the NLRP3 Inflammasome Pathway Are Involved in the Anti-inflammatory Effect of Anthocyanin}

Evidence shows that NF- $\kappa \mathrm{B}$ and the NLRP3 inflammasome pathway play an important role in the inflammatory signaling pathways (Dolunay et al., 2017). To identify whether NF-кB and NLRP3 are also involved in the anthocyanin-induced antiinflammatory effect following $\mathrm{MCAO} / \mathrm{R}$, we performed western blot analysis. As shown in Figure 5, anthocyanin reduced the expression of $\mathrm{p}-\mathrm{NF}-\kappa \mathrm{B}$ p50, $\mathrm{p}-\mathrm{NF}-\kappa \mathrm{B}$ p $65, \mathrm{p}-\mathrm{I} \kappa \mathrm{B}$, and NLRP3 following MCAO/R. Based on the obtained results, the mechanisms of action and the underlying signaling pathways of anthocyanin are proposed in Figure 6.

\section{DISCUSSION}

Our study demonstrated that the administration of anthocyanin significantly reduced infarct volume, neurological scores, cognitive function decline, cerebral edema and the concentration of $\mathrm{EB}$ in $\mathrm{MCAO} / \mathrm{R}$ rats by inhibiting MMP-9, Bcl-2 family and inflammation-related molecules including TNF- $\alpha$, IL-1 $\beta$, and IL-6. In addition, NF- $\mathrm{B}$ 


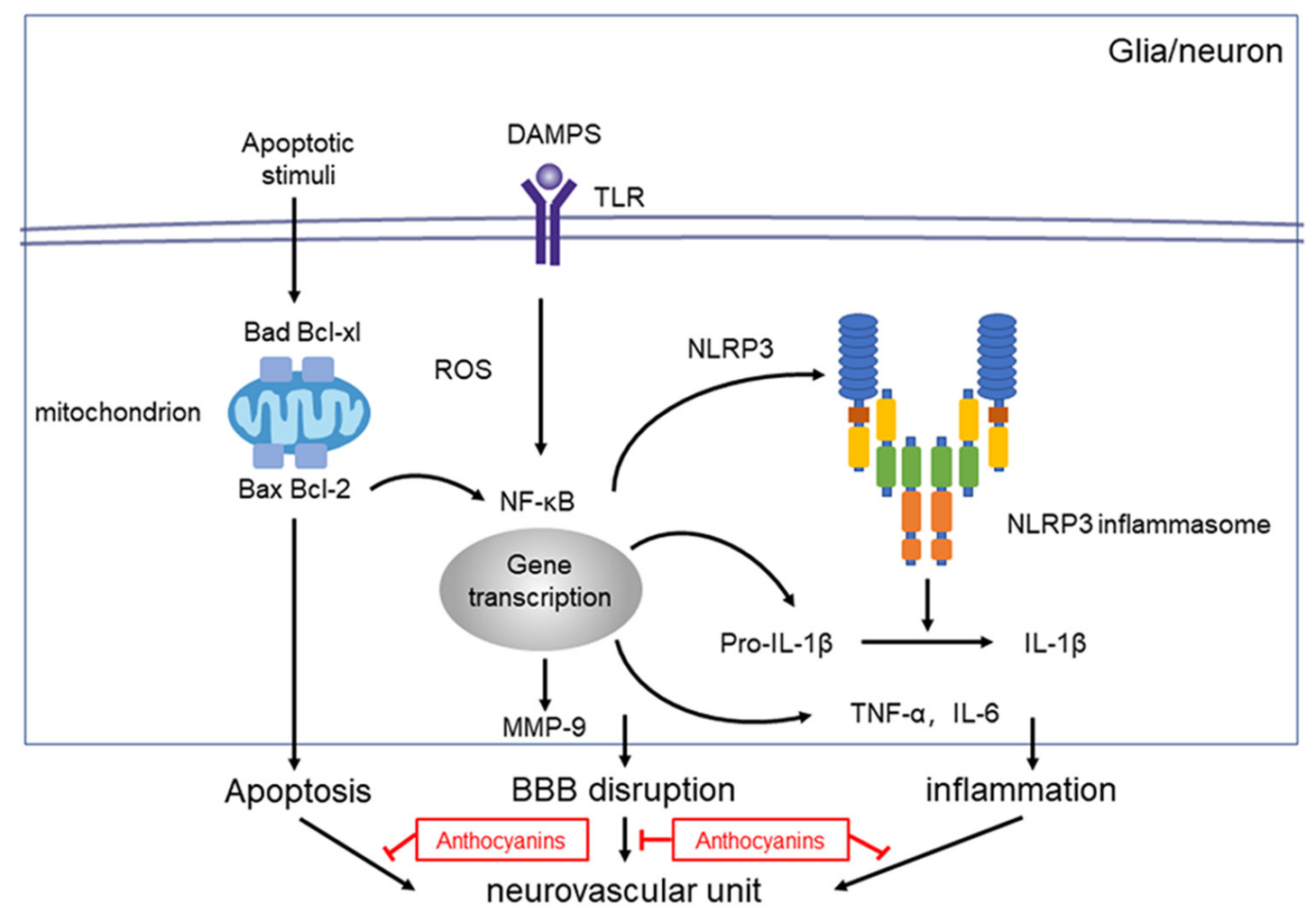

FIGURE 6 | Proposed mechanisms of anthocyanin's effect on NVU dysfunction after ischemic stroke. Injury to the neurovascular unit results in either death or injury to endothelial cells, neurons, microglia etc. Following hypoxia and ischemia, reactive oxygen species (ROS) are generated and NF-кB are activated, which activates the NLRP3 inflammasome pathway, and triggers the expression of IL-1 $\beta$. Anthocyanin ameliorates the ischemia-hypoxia-induced BBB disruption by down regulating the expression of MMP-9. Anthocyanin down-regulate the expression level of Bax/Bcl-2 and Bad/Bcl-xl, which ultimately inhibits the extrinsic and intrinsic apoptotic signaling pathways to block apoptosis.

and the NLRP3 inflammasome pathway may play a role in the anti-inflammatory effect of anthocyanin. Taken together, these results may enhance our understanding of the NVU protection properties of anthocyanin against ischemic stroke through multiple pathways and provide an alternative option for the future prevention and treatment of stroke.

Stroke is one of the most common causes of death worldwide and is a major cause of acquired disability in adults, with highly complex pathological process (Soler and Ruiz, 2010). In recent years, the NVU has become a new target for stroke treatment ( $\mathrm{Hu}$ et al., 2017). NVU is composed of neurons, the BBB, glial cells (astrocytes, microglia), vascular cells (endothelial cells), and the ECM. The BBB is the core structure of the NVU, and matrix metalloproteinases (MMPs) have been widely considered to play a key role in the disruption of the BBB following stroke (Liu et al., 2012). In the early phase of stroke, MMPs cause multiple NVU dysfunctions, such as neuronal death and BBB leakage (Rosell and Lo, 2008).

Previous studies have shown that MMP-9 is abnormally expressed in brains suffering from cerebral ischemic injury and that it promotes brain damage and BBB breakdown (Rosenberg and Yang, 2007). In humans, patients with ischemic stroke show higher levels of MMP-9 in the blood, and more importantly, MMP-9 levels are associated with a poorer prognosis (Dejonckheere et al., 2011). Specifically, inhibitors or gene suppression of MMP-9, significantly reduced infarct size and bleeding complications (Hu et al., 2009). Our results indicate that anthocyanin may protect BBB permeability through the inhibition MMP-9. Consistent with our results, studies that administered MMP inhibitors prior to stroke showned similar benefits (Asahi et al., 2000).

Blood-brain barrier dysfunction caused by apoptosis and inflammation, is a key factor that causes NVU injury, which promotes the development of ischemic brain injury (Lakhan et al., 2009). Apoptosis contributes to a significant proportion of neuronal death that occurs following brain ischemia (Radak et al., 2017). The Bcl-2 protein family is composed of pro-apoptotic (Bax, Bad) and anti-apoptotic (Bcl-2, Bcl-xl) members, which are major regulators of the mitochondrial apoptotic pathway (Gross, 2016), and are involved in the occurrence and development of stroke (Qi et al., 2015). In this study, anthocyanin alleviated apoptosis injury resulting from $\mathrm{MCAO} / \mathrm{R}$. Additionally, anthocyanin increased levels of $\mathrm{Bcl}-2$ and $\mathrm{Bcl}-\mathrm{xl}$ in $\mathrm{MCAO} / \mathrm{R}$ rats, and, it decreased levels of $\mathrm{Bax}$ and Bad. 
This finding suggests that anthocyanin can protect the NVU by inhibiting apoptosis, and therefore, may be a potential candidate for the treatment of ischemia-reperfusion injury.

After stroke, cerebral ischemia injury induces a robust inflammatory response, causing great damage to the BBB. Damage-associated molecular patterns (DAMPs) are the main signals that trigger inflammatory reactions. Most DAMPs, including HMGB-1 or heat shock proteins, are released from dying and dead cells (Chen and Nunez, 2010), and are sensed by pattern recognition receptors, including Toll-like receptors (TLRs) and scavenger receptors. Once the DAMP-receptor signaling is activated, inflammatory mediators such as cytokines (IL-1 $\beta$, TNF- $\alpha$, and IL-6), chemokines and reactive oxygen species (ROS) are released from the NVU component cells to exacerbate cell death and lead to BBB breakdown (Macrez et al., 2011). The NF-кB transcriptional activation pathway is considered to be a master regulator of inflammation and is indeed critical to the regulation of apoptosis, and play a key role in stroke (Harari and Liao, 2010). Decreasing NF- $\kappa$ B expression could reduce stroke size, edema, and neurological deficits in the rat permanent MCAO model (Wang et al., 2010). Furthermore, NF- $\kappa$ B can regulate the expression of MMP-9 (Wang et al., 2009). Inhibitors of MMP-9 can significantly reduce cerebral edema and BBB disruption in stroke (Wang et al., 2009). Our results showed that anthocyanin significantly decreased the expression

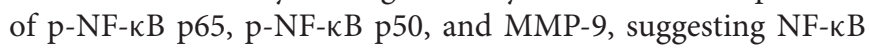
pathway is involved in the NVU protection effect of anthocyanin.

One study found that the expression of NLRP3 was increased in the brain tissue of stroke patients (Fann et al., 2013). The activation of TLR-4 activates NF- $\kappa \mathrm{B}$ during cerebral ischemia, and NF- $\kappa \mathrm{B}$ further promotes the production of NLRP3 and IL-1 $\beta$ - thus, enhancing inflammation (Minutoli et al., 2016). The NLRP3 inflammasome has become a critical mediator of post-ischemic inflammation, which eventually leads to cell death (Yang et al., 2014). NLRP3 was expressed in the neurons, microglia, and endothelial cells of ischemic brains, all of which primarily make up the NVU (Famakin, 2014). In primary cortical neurons, ischemic conditions increased NLRP3 expression and IL-1 $\beta$ levels. Similarly, levels of NLRP3 and IL-1 $\beta$ were elevated in the brains of I/R mice (Fann et al., 2013). In addition, studies have shown that in a mouse MCAO model, NLRP3 ${ }^{-/-}$mice exhibited reduced infarct volumes, decreased edema formation, and preserved BBB permeability (Fernandez-Cadenas et al., 2012; Lambertsen et al., 2012). In this study, our results showed that anthocyanin could inhibit MCAO/R-induced up regulation of NLRP3 in the ischemic brains of rats, indicating that anthocyanin protects the NVU against inflammation, at least in part, through a mechanism mediated by NLRP3 signaling.

Anthocyanins, which are flavonoids formed by anthocyanidins and sugars through glycosidic bonds, are natural antioxidants with a wide range of biomedical functions. Anthocyanins are thought to benefit cardiovascular, inflammatory, and degenerative diseases (Zafra-Stone et al., 2007). Anthocyanins are recognized as ROS scavengers, and endogenous ROS are mainly produced by the mitochondrial respiratory chain and the NADPH oxidase system (Dikalov, 2011). Mitochondria and NADPH oxidase have previously been implicated in BBB dysfunction and its loss of integrity (Doll et al., 2015; Schiavone et al., 2017). Studies have shown that anthocyanins have pharmacological effects on mitochondria cardioprotection through the following mechanisms: reduction of cytosolic cytochrome c preventing apoptosis and sustainment of electron transfer between NADH dehydrogenase and cytochrome c supporting oxidative phosphorylation in ischemiadamaged mitochondria (Liobikas et al., 2016). However, further research is required to investigate the role of different free radical sources in the inhibition of cerebral ischemia by anthocyanins.

Although anthocyanins have considerable beneficial effects, we still need to pay attention to the safety of anthocyanins. Anthocyanins do not appear to be readily absorbed or metabolized. A weight-of-evidence analysis indicates that anthocyanins are not genotoxic (Haveland-Smith, 1981; Ferguson et al., 1985). The acute oral toxicity is low; for instance, the oral $\mathrm{LD}_{50}$ for a combination of the glycosides of cyanidin, delphinidin, petundin, and malvidin was more than $25 \mathrm{~g} / \mathrm{kg}$ for mice and more than $20 \mathrm{~g} / \mathrm{kg}$ for rats (Hallagan et al., 1995). However, no study gives the lethal dose of anthocyanins in the case of intraperitoneal injection. In our study, the anthocyanin were intraperitoneally injected into rats by the concentrations of 50,100 , and $200 \mathrm{mg} / \mathrm{kg}$. It may be concluded by the oral studies that intraperitoneal injection of anthocyanins may be safe in a relatively lower dose, and some other scholars have reported the benefits of intraperitoneal injection of anthocyanins in other doses (Rossi et al., 2003; Matsumoto et al., 2006; Khan et al., 2016). Despite all of these, we still need to focus some sights to the intraperitoneal injection security issues of anthocyanins, and further research is required to investigate the safe dosage of intraperitoneal injection of anthocyanins.

In addition, the anthocyanin attenuated progression of cerebral ischemic damage in rats was, associated with the suppression of the JNK and p53 signaling pathway (Shin et al., 2006). Our study found that anthocyanin could also inhibit MMP-9 and NLRP3 levels to facilitate NVU protection. Therefore, anthocyanin may potentially be a safe and effective treatment for improving stroke through multipleeffects.

Taken together, the NVU is important in stroke pathophysiology, and the investigation of multiple-pathways that protect of the NVU may lead to novel approaches for this devastating disease. Our study found that anthocyanin could inhibit apoptosis and inflammation to protect the NVU in rats impaired by MCAO/R. Additionally, NF- $\mathrm{B}, \mathrm{MMP}-9$, and the NLRP3 inflammasome pathway might be involved anthocyanin's protective effects in the NVU. Therefore, anthocyanin could protect the NVU through multiple pathways, and might be a potential candidate for the prevention and treatment of stroke.

\section{DATA AVAILABILITY STATEMENT}

Data and materials will be available from the authors upon reasonable request and with the permission of $\mathrm{ZP}$ after submission of the thesis. 


\section{AUTHOR CONTRIBUTIONS}

ZP carried out the experiments and assisted with data analysis. MC and GD helped with the manuscript preparation and the in vivo experiments. TY helped with the design of the study. YL conducted the RT-PCR experiment and assisted with the manuscript preparation. TJ performed the Western Blot experiment and assisted with the data analysis. YP helped conduct the experiments and contributed to the writing of the manuscript.

\section{REFERENCES}

Asahi, M., Asahi, K., Wang, X., and Lo, E. H. (2000). Reduction of tissue plasminogen activator-induced hemorrhage and brain injury by free radical spin trapping after embolic focal cerebral ischemia in rats. J. Cereb. Blood Flow Metab. 20, 452-457. doi: 10.1097/00004647-200003000-00002

Chen, G. Y., and Nunez, G. (2010). Sterile inflammation: sensing and reacting to damage. Nat. Rev. Immunol. 10, 826-837. doi: 10.1038/nri2873

Dejonckheere, E., Vandenbroucke, R. E., and Libert, C. (2011). Matrix metalloproteinases as drug targets in ischemia/reperfusion injury. Drug Discov. Today 16, 762-778. doi: 10.1016/j.drudis.2011.06.009

Dikalov, S. (2011). Cross talk between mitochondria and NADPH oxidases. Free Radic. Biol. Med. 51, 1289-1301. doi: 10.1016/j.freeradbiomed.2011.06.033

Doll, D. N., Hu, H., Sun, J., Lewis, S. E., Simpkins, J. W., and Ren, X. (2015). Mitochondrial crisis in cerebrovascular endothelial cells opens the blood-brain barrier. Stroke 46, 1681-1689. doi: 10.1161/STROKEAHA.115.009099

Dolunay, A., Senol, S. P., Temiz-Resitoglu, M., Guden, D. S., Sari, A. N., SahanFirat, S., et al. (2017). Inhibition of NLRP3 inflammasome prevents lps-induced inflammatory hyperalgesia in mice: contribution of NF-kappaB, caspase-1/11, ASC, NOX, and NOS Isoforms. Inflammation 40, 366-386. doi: 10.1007/ s10753-016-0483-3

Elmore, S. (2007). Apoptosis: a review of programmed cell death. Toxicol. Pathol. 35, 495-516. doi: 10.1080/01926230701320337

Famakin, B. M. (2014). The immune response to acute focal cerebral ischemia and associated post-stroke immunodepression: a focused review. Aging Dis. 5, 307-326. doi: 10.14336/AD.2014.0500307

Fann, D. Y., Lee, S. Y., Manzanero, S., Tang, S. C., Gelderblom, M., Chunduri, P., et al. (2013). Intravenous immunoglobulin suppresses NLRP1 and NLRP3 inflammasome-mediated neuronal death in ischemic stroke. Cell Death Dis. 4:e790. doi: 10.1038/cddis.2013.326

Fann, D. Y., Lim, Y. A., Cheng, Y. L., Lok, K. Z., Chunduri, P., Baik, S. H., et al. (2017). Evidence that NF-кB and MAPK signaling promotes NLRP inflammasome activation in neurons following ischemic stroke. Mol. Neurobiol. 55, 1082-1096. doi: 10.1007/s12035-017-0394-9

Ferguson, L. R., van Zijl, P., Holloway, W. D., and Jones, W. T. (1985). Condensed tannins induce micronuclei in cultured V79 Chinese hamster cells. Mutat. Res. 158, 89-95. doi: 10.1016/0165-1218(85)90102-8

Fernandez-Cadenas, I., Del Rio-Espinola, A., Giralt, D., Domingues-Montanari, S., Quiroga, A., Mendioroz, M., et al. (2012). IL1B and VWF variants are associated with fibrinolytic early recanalization in patients with ischemic stroke. Stroke 43, 2659-2665. doi: 10.1161/STROKEAHA.112.657007

Gao, L., Dong, Q., Song, Z., Shen, F., Shi, J., and Li, Y. (2017). NLRP3 inflammasome: a promising target in ischemic stroke. Inflamm. Res. 66, 17-24. doi: 10.1007/s00011-016-0981-7

Gross, A. (2016). BCL-2 family proteins as regulators of mitochondria metabolism. Biochim. Biophys. Acta 1857, 1243-1246. doi: 10.1016/j.bbabio.2016. 01.017

Hallagan, J. B., Allen, D. C., and Borzelleca, J. F. (1995). The safety and regulatory status of food, drug and cosmetics colour additives exempt from certification. Food Chem. Toxicol. 33, 515-528. doi: 10.1016/0278-6915(95)00010-Y

Harari, O. A., and Liao, J. K. (2010). NF-kappaB and innate immunity in ischemic stroke. Ann. N. Y. Acad. Sci. 1207, 32-40. doi: $10.1111 / \mathrm{j} .1749-6632.2010 .05735 . \mathrm{x}$

\section{FUNDING}

This study was supported by the National Nature Science Foundation of China (No. 81373534).

\section{ACKNOWLEDGMENTS}

The authors thank Dr. Liqun Pan at the Affiliated Hospital of Nanjing University of Chinese Medicine (Nanjing, China) for providing necessary facilities.

Haveland-Smith, R. B. (1981). Evaluation of the genotoxicity of some natural food colours using bacterial assays. Mutat. Res. 91, 285-290. doi: 10.1016/01657992(81)90002-6

Hu, Q., Chen, C., Yan, J., Yang, X., Shi, X., Zhao, J., et al. (2009). Therapeutic application of gene silencing MMP-9 in a middle cerebral artery occlusioninduced focal ischemia rat model. Exp. Neurol. 216, 35-46. doi: 10.1016/j. expneurol.2008.11.007

Hu, X., De Silva, T. M., Chen, J., and Faraci, F. M. (2017). Cerebral vascular disease and neurovascular injury in ischemic stroke. Circ. Res. 120, 449-471. doi: 10.1161/CIRCRESAHA.116.308427

Khan, M. S., Ali, T., Kim, M. W., Jo, M. H., Jo, M. G., Badshah, H., et al. (2016). Anthocyanins protect against LPS-induced oxidative stressmediated neuroinflammation and neurodegeneration in the adult mouse cortex. Neurochem. Int. 100, 1-10. doi: 10.1016/j.neuint.2016.08.005

Kurzepa, J., Kurzepa, J., Golab, P., Czerska, S., and Bielewicz, J. (2014). The significance of matrix metalloproteinase (MMP)-2 and MMP-9 in the ischemic stroke. Int. J. Neurosci. 124, 707-716. doi: 10.3109/00207454.2013.872102

Lake, E. M., Bazzigaluppi, P., Mester, J., Thomason, L. A., Janik, R., Brown, M., et al. (2017). Neurovascular unit remodelling in the subacute stage of stroke recovery. Neuroimage 146, 869-882. doi: 10.1016/j.neuroimage.2016.09.016

Lakhan, S. E., Kirchgessner, A., and Hofer, M. (2009). Inflammatory mechanisms in ischemic stroke: therapeutic approaches. J. Transl. Med. 7:97. doi: 10.1186/ 1479-5876-7-97

Lambertsen, K. L., Biber, K., and Finsen, B. (2012). Inflammatory cytokines in experimental and human stroke. J. Cereb. Blood Flow Metab. 32, 1677-1698. doi: $10.1038 / \mathrm{jcbfm} .2012 .88$

Li, D., Wang, P., Luo, Y., Zhao, M., and Chen, F. (2017). Health benefits of anthocyanins and molecular mechanisms: update from recent decade. Crit. Rev. Food Sci. Nutr. 57, 1729-1741. doi: 10.1080/10408398.2015.1030064

Li, Y., Chen, J., Chen, X. G., Wang, L., Gautam, S. C., Xu, Y. X., et al. (2002). Human marrow stromal cell therapy for stroke in rat: neurotrophins and functional recovery. Neurology 59, 514-523. doi: 10.1212/WNL.59.4.514

Lin, T. N., He, Y. Y., Wu, G., Khan, M., and Hsu, C. Y. (1993). Effect of brain edema on infarct volume in a focal cerebral ischemia model in rats. Stroke 24, 117-121. doi: 10.1161/01.STR.24.1.117

Liobikas, J., Skemiene, K., Trumbeckaite, S., and Borutaite, V. (2016). Anthocyanins in cardioprotection: a path through mitochondria. Pharmacol. Res. 113, 808-815. doi: 10.1016/j.phrs.2016.03.036

Liu, J., Jin, X., Liu, K. J., and Liu, W. (2012). Matrix metalloproteinase2-mediated occludin degradation and caveolin-1-mediated claudin-5 redistribution contribute to blood-brain barrier damage in early ischemic stroke stage. J. Neurosci. 32, 3044-3057. doi: 10.1523/JNEUROSCI.6409-11. 2012

Longa, E. Z., Weinstein, P. R., Carlson, S., and Cummins, R. (1989). Reversible middle cerebral artery occlusion without craniectomy in rats. Stroke 20, 84-91. doi: 10.1161/01.STR.20.1.84

Macrez, R., Ali, C., Toutirais, O., Le Mauff, B., Defer, G., Dirnagl, U., et al. (2011). Stroke and the immune system: from pathophysiology to new therapeutic strategies. Lancet Neurol. 10, 471-480. doi: 10.1016/S1474-4422(11)70066-7

Maki, T., Hayakawa, K., Pham, L. D., Xing, C., Lo, E. H., and Arai, K. (2013). Biphasic mechanisms of neurovascular unit injury and protection in CNS diseases. CNS Neurol Disord. Drug Targets 12, 302-315. doi: 10.2174/ 1871527311312030004 
Matsumoto, H., Nakamura, Y., Iida, H., Ito, K., and Ohguro, H. (2006). Comparative assessment of distribution of blackcurrant anthocyanins in rabbit and rat ocular tissues. Exp. Eye Res. 83, 348-356. doi: 10.1016/j.exer.2005. 12.019

Minutoli, L., Puzzolo, D., Rinaldi, M., Irrera, N., Marini, H., Arcoraci, V., et al. (2016). ROS-Mediated NLRP3 Inflammasome Activation in Brain, Heart, Kidney, and Testis Ischemia/Reperfusion Injury. Oxid. Med. Cell. Longev. 2016:2183026. doi: 10.1155/2016/2183026

Qi, Z., Dong, W., Shi, W., Wang, R., Zhang, C., Zhao, Y., et al. (2015). Bcl-2 phosphorylation triggers autophagy switch and reduces mitochondrial damage in limb remote ischemic conditioned rats after ischemic stroke. Transl. Stroke Res. 6, 198-206. doi: 10.1007/s12975-015-0393-y

Radak, D., Katsiki, N., Resanovic, I., Jovanovic, A., Sudar-Milovanovic, E., Zafirovic, S., et al. (2017). Apoptosis and acute brain ischemia in ischemic stroke. Curr. Vasc. Pharmacol. 15, 115-122. doi: 10.2174/ 1570161115666161104095522

Rosell, A., and Lo, E. H. (2008). Multiphasic roles for matrix metalloproteinases after stroke. Curr. Opin. Pharmacol. 8, 82-89. doi: 10.1016/j.coph.2007.12. 001

Rosenberg, G. A. (2012). Neurological diseases in relation to the blood-brain barrier. J. Cereb. Blood Flow Metab. 32, 1139-1151. doi: 10.1038/jcbfm. 2011.197

Rosenberg, G. A., and Yang, Y. (2007). Vasogenic edema due to tight junction disruption by matrix metalloproteinases in cerebral ischemia. Neurosurg. Focus 22:E4. doi: 10.3171/foc.2007.22.5.5

Rossi, A., Serraino, I., Dugo, P., Di Paola, R., Mondello, L., Genovese, T., et al. (2003). Protective effects of anthocyanins from blackberry in a rat model of acute lung inflammation. Free Radic. Res. 37, 891-900. doi: 10.1080/ 1071576031000112690

Schiavone, S., Mhillaj, E., Neri, M., Morgese, M. G., Tucci, P., Bove, M., et al. (2017). Early loss of blood-brain barrier integrity precedes NOX2 elevation in the prefrontal cortex of an animal model of psychosis. Mol. Neurobiol. 54, 2031-2044. doi: 10.1007/s12035-016-9791-8

Shin, W. H., Park, S. J., and Kim, E. J. (2006). Protective effect of anthocyanins in middle cerebral artery occlusion and reperfusion model of cerebral ischemia in rats. Life Sci. 79, 130-137. doi: 10.1016/j.lfs.2005.12.033

Soler, E. P., and Ruiz, V. C. (2010). Epidemiology and risk factors of cerebral ischemia and ischemic heart diseases: similarities and differences. Curr. Cardiol. Rev. 6, 138-149. doi: 10.2174/157340310791658785

Tong, Y., Ding, Z. H., Zhan, F. X., Cai, L., Yin, X., Ling, J. L., et al. (2015). The NLRP3 inflammasome and stroke. Int. J. Clin. Exp. Med. 8, 4787-4794.
Turner, R. J., and Sharp, F. R. (2016). Implications of MMP9 for blood brain barrier disruption and hemorrhagic transformation following ischemic stroke. Front. Cell Neurosci. 10:56. doi: 10.3389/fncel.2016.00056

Vidale, S., Consoli, A., Arnaboldi, M., and Consoli, D. (2017). Postischemic inflammation in acute stroke. J. Clin. Neurol. 13, 1-9. doi: 10.3988/jcn.2017. 13.1.1

Wang, E., Yin, Y., Xu, C., and Liu, J. (2014). Isolation of high-purity anthocyanin mixtures and monomers from blueberries using combined chromatographic techniques. J. Chromatogr. A 1327, 39-48. doi: 10.1016/j.chroma.2013. 12.070

Wang, G., Guo, Q., Hossain, M., Fazio, V., Zeynalov, E., Janigro, D., et al. (2009). Bone marrow-derived cells are the major source of MMP-9 contributing to blood-brain barrier dysfunction and infarct formation after ischemic stroke in mice. Brain Res. 1294, 183-192. doi: 10.1016/j.brainres.2009.07.070

Wang, L., Zhang, X., Liu, L., Yang, R., Cui, L., and Li, M. (2010). Atorvastatin protects rat brains against permanent focal ischemia and downregulates HMGB1, HMGB1 receptors (RAGE and TLR4), NF-kappaB expression. Neurosci. Lett. 471, 152-156. doi: 10.1016/j.neulet.2010.01.030

Wang, Q., van Hoecke, M., Tang, X. N., Lee, H., Zheng, Z., Swanson, R. A., et al. (2009). Pyruvate protects against experimental stroke via an anti-inflammatory mechanism. Neurobiol. Dis. 36, 223-231. doi: 10.1016/j.nbd.2009.07.018

Watts, L. T., Lloyd, R., Garling, R. J., and Duong, T. (2013). Stroke neuroprotection: targeting mitochondria. Brain Sci. 3, 540-560. doi: 10.3390/brainsci3020540

Yang, F., Wang, Z., Wei, X., Han, H., Meng, X., Zhang, Y., et al. (2014). NLRP3 deficiency ameliorates neurovascular damage in experimental ischemic stroke. J. Cereb. Blood Flow Metab. 34, 660-667. doi: 10.1038/jcbfm.2013.242

Zafra-Stone, S., Yasmin, T., Bagchi, M., Chatterjee, A., Vinson, J. A., and Bagchi, D. (2007). Berry anthocyanins as novel antioxidants in human health and disease prevention. Mol. Nutr. Food Res. 51, 675-683. doi: 10.1002/mnfr.200700002

Conflict of Interest Statement: The authors declare that the research was conducted in the absence of any commercial or financial relationships that could be construed as a potential conflict of interest.

Copyright (C) 2018 Pan, Cui, Dai, Yuan, Li, Ji and Pan. This is an open-access article distributed under the terms of the Creative Commons Attribution License (CC BY). The use, distribution or reproduction in other forums is permitted, provided the original author(s) and the copyright owner(s) are credited and that the original publication in this journal is cited, in accordance with accepted academic practice. No use, distribution or reproduction is permitted which does not comply with these terms. 